\title{
El equipo corazón-cerebro: neurocardiología
}

\author{
The heart-brain team: neurocardiology
}

Amado Jiménez-Ruiz" ${ }^{*}$, José L. Ruiz-Sandoval², Diego Araiza-Garaygordobiß ${ }^{3}$ y Daniel Sierra-Lara ${ }^{3}$

${ }^{1}$ Stroke, Dementia \& Heart Disease Lab, Western University, Ontario, Canadá; ${ }^{2}$ Servicio de Neurología de Neurología, Hospital Civil Fray Antonio Alcalde, Guadalajara, Jalisco, México; ${ }^{3}$ Departamento de Cardiología, Instituto Nacional de Cardiología Ignacio Chávez, Ciudad de México, México

Las enfermedades cardiovasculares y cerebrovasculares ocupan los primeros lugares de morbimortalidad en México y el mundo. El sistema nervioso y el sistema cardiovascular comparten una red intrínseca de mecanismos fisiológicos y patológicos que competen al neurólogo y al cardiólogo. La neurocardiología abarca trastornos cardiacos que afectan al cerebro, trastornos cerebrales que afectan al corazón y enfermedades sistémicas que afectan a ambos órganos. La enfermedad cerebrovascular es la causa más importante de discapacidad, la segunda causa de deterioro cognitivo y la primera causa de epilepsia en el aduto mayor.

A continuación presentamos algunos ejemplos que ayudan a entender las posibles implicaciones entre ambos sistemas (Fig. 1).

\section{Cardioembolismo cerebral}

Este mecanismo corresponde a un $25 \%$ de todos los infartos cerebrales, en especial en el contexto de fibrilación auricular (FA), generando una alta morbimortalidad sobre todo en población geriátrica. Los infartos cerebrales relacionados con FA tienden a ser más graves y discapacitantes que los debidos a otros mecanismos $^{1,2}$. Recientemente se han descrito dos conceptos que cuestionan lo que creíamos saber: el primero es que no es necesaria la presencia de FA para

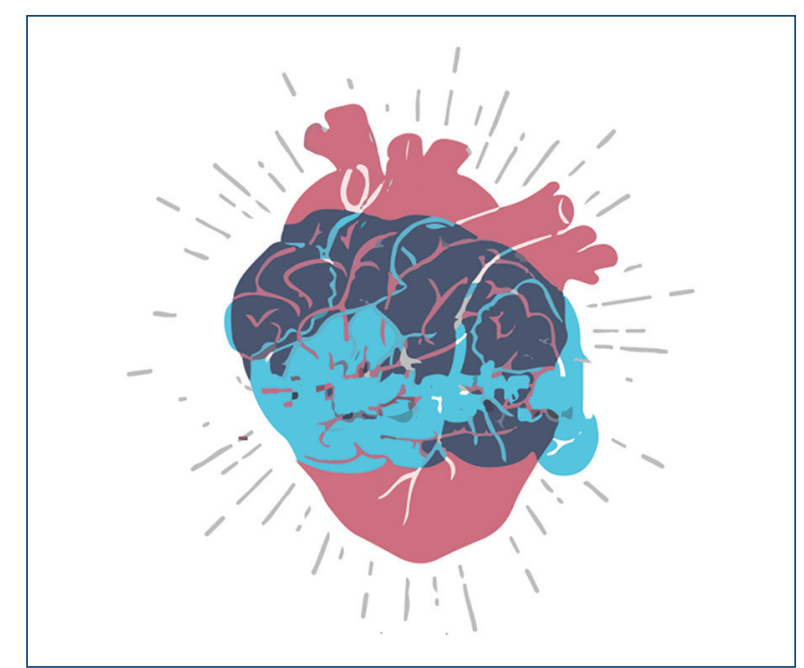

Figura 1. Corazón-cerebro.

generar trombosis intracavitaria; y el segundo, que la FA puede tener un origen cardiaco, cerebral o mixto. La aurícula izquierda sufre cambios eléctricos, estructurales y funcionales que pueden iniciar la trombogénesis, aun en ausencia de una arritmia demostrable. A este proceso se le conoce como cardiopatía auricular y abre una nueva línea de investigación en factores inflamatorios e inmunitarios de disfunción miocárdica, aun en ausencia de arritmias demostrables ${ }^{3}$.
Correspondencia:

*Amado Jiménez-Ruiz

E-mail: dr.amadojimenez@gmail.com
Fecha de recepción: 11-05-2020

Fecha de aceptación: 06-10-2020 DOI: 10.24875/ACM.20000213
Disponible en internet: 02-07-2021 Arch Cardiol Mex. 2021;91(3):391-393 www.archivoscardiologia.com 1405-9940 / @ 2020 Instituto Nacional de Cardiología Ignacio Chávez. Publicado por Permanyer. Este es un artículo open access bajo la licencia CC BY-NC-ND (http://creativecommons.org/licenses/by-nc-nd/4.0/). 
Los infartos insulares son una prueba de que el control del sistema nervioso autonómico influye en la modulación del control cardiaco, causando alteraciones de la frecuencia y arritmias. En estos casos, la FA podría ser una causa directa de daño cerebral, o neurogénica, mientras que la que se detecta antes del infarto cerebral podría ser una causa directa de daño miocárdico, o cardiogénica. Hacer la distinción podría influir en la evolución natural de la enfermedad y en su pronóstico ${ }^{4}$.

\section{Enfermedades sistémicas}

Las enfermedades autoinmunitarias, como el lupus eritematoso generalizado, con frecuencia cursan con disfunción endotelial expresada como vasculitis en diferentes órganos (incluyendo el corazón y el cerebro), y conforman una de las principales causas de morbimortalidad en esta población ${ }^{5,6}$.

Las enfermedades mitocondriales son otro ejemplo de enfermedades sistémicas que causan alteraciones cardiacas y neurológicas, con el común denominador de falla energética celular ${ }^{7,8}$.

Las principales causas de paro cardiaco súbito son la cardiopatía isquémica, las cardiomiopatías y los trastornos primarios del ritmo cardiaco ${ }^{9}$.

Los avances en los protocolos prehospitalarios de soporte cardiaco avanzado han aumentado el número de sobrevivientes de paro cardiaco en las unidades de cuidados intensivos, y las últimas guías incluyen recomendaciones para ventilación mecánica, sedación e hipotermia inducida en las primeras 6 horas después del evento ${ }^{10}$.

\section{Angiopatía amiloide, fibrilación auricular y deterioro cognitivo}

Con el envejecimiento de la población cada vez es más común encontrar pacientes geriátricos con FA y angiopatía amiloide. Esta última es causada por el depósito de $\beta$-amiloide en los vasos y en el parénquima cerebral. Clínicamente se manifiesta por deterioro cognitivo y hemorragia intracerebral recurrente, sobre todo en pacientes que toman anticoagulantes orales. A pesar de esta asociación, no se incluye dentro de los algoritmos diagnósticos y escalas de riesgo actuales. Determinar la presencia y la carga lesional de angiopatía amiloide previamente a iniciar la anticoagulación oral para FA podría ser un paso importante para prevenir la hemorragia intracerebral ${ }^{11}$.
Las mutaciones en los genes responsables de la Enfermedad de Alzheimer familiar (PSEN 1, PSEN 2 y APP) y el posterior depósito de proteína $\beta$-amiloide podrían explicar parte del vínculo entre el envejecimiento cardiaco, el desarrollo de insuficiencia cardiaca y la aparición de enfermedades neurodegenerativas como un modelo de enfermedad multisistémica. ${ }^{12,13}$. Existe amplia evidencia observacional que asocia la propia FA con deterioro cognitivo, tanto para el tipo vascular como para la enfermedad de Alzheimer. Los posibles mecanismos incluyen infarto cerebral clínico o silente, hemorragia intracerebral, hipoperfusión global y el aumento en el depósito de $\beta$-amiloide cerebra $^{14}$. La anticoagulación podría reducir el riesgo de deterioro cognitivo en estos pacientes ${ }^{15}$.

La enfermedad vascular cerebral coexiste en la mayoría de las enfermedades neurodegenerativas que cursan con deterioro cognitivo, y hoy en día es el único componente tratable y potencialmente prevenible de estos trastornos ${ }^{16}$.

\section{Hipertensión arterial}

La hipertensión arterial sistémica es el factor de riesgo cardiovascular modificable más importante para el cardiólogo y el neurólogo. La hemorragia intracerebral hipertensiva es la primera causa de hemorragia intracerebral en México y en el mundo. La hipertensión se asocia a enfermedad de pequeño vaso, incluyendo enfermedad de sustancia blanca, infarto cerebral lacunar, microhemorragias y deterioro cognitivo. El estudio SPRINT MIND demostró (mediante seguimiento neuropsicológico y estudios de imagen cerebral) que el control intensivo de la presión arterial disminuye el riesgo de deterioro cognitivo leve, y extendiendo este seguimiento a largo plazo podría disminuir el riesgo de trastorno neurocognitivo mayor (antes demencia) ${ }^{17}$.

\section{Dislipidemia}

La dislipidemia, sobre todo en función de las lipoproteínas de baja densidad, es una piedra angular de la prevención primaria y secundaria de eventos cardiovasculares. Existe evidencia de que los niveles muy bajos podrían asociarse a hemorragia intracerebral, sobre todo en las mujeres ${ }^{18}$, y este factor de riesgo tampoco se encuentra estratificado dentro de los algoritmos diagnósticos y terapeúticos actuales. Sin embargo, un metaanálisis concluye que los beneficios de la terapia de reducción de lípidos en la prevención del infarto cerebral superan en gran medida el riesgo de 
hemorragia intracerebral, y no debe disuadir a los médicos de tratar este trastorno metabólico ${ }^{19}$.

\section{Hemorragia subaracnoidea}

La mayoría de los pacientes con hemorragia subaracnoidea aneurismática presentan alteraciones electrocardiográficas, así como elevación de troponinas. La muerte súbita, el miocardio aturdido y la miocardiopatía inducida por estrés (síndrome de Takotsubo) se atribuyen a una tormenta de catecolaminas, por daño al sistema nervioso.

\section{Otros ejemplos}

- Protección embólica en procedimientos tales como el implante valvular aórtico transcatéter (TAVI, transcatheter aortic valve implantation) y otras complicaciones cerebrales de procedimientos cardiacos.

- Dispositivos implantables para detección de fibrilación auricular oculta posterior al infarto cerebral.

- Infarto cerebral, embolia paradójica, migraña y foramen oval permeable.

- Biomarcadores en el diagnóstico de enfermedad cardiocerebral (troponinas, péptido natriurético, dímero D).

- Factores pronósticos de la encefalopatía hipóxico-isquémica tras paro cardiaco y diagnóstico de muerte cerebral.

- Diagnóstico y tratamiento de trastornos del ritmo cardiaco en pacientes con enfermedades sistémicas ( $p$. ej., distrofias musculares y ataxias de origen genético)

\section{Conclusión}

Existen muchos vínculos entre la neurología y la cardiología. Es necesario integrar a ambos profesionales en equipos corazón-cerebro que participen en la prevención, el diagnóstico y el tratamiento de las enfermedades que causan más morbimortalidad en nuestro país ${ }^{20}$.

\section{Agradecimientos}

Agradecemos a la Lic. Almendra Covarrubias por la creación de la imagen figura 1.

\section{Financiamiento}

La presente investigación no ha recibido ninguna beca específica de agencias de los sectores público, comercial o sin ánimo de lucro.

\section{Conflicto de intereses}

Ninguno.

\section{Responsabilidades éticas}

Protección de personas y animales. Los autores declaran que para esta investigación no se han realizado experimentos en seres humanos ni en animales.

Confidencialidad de los datos. Los autores declaran que han seguido los protocolos de su centro de trabajo sobre la publicación de datos de pacientes.

Derecho a la privacidad y consentimiento informado. Los autores declaran que en este artículo no aparecen datos de pacientes.

\section{Bibliografía}

1. Griñán K, Arboix A, Massons J, Díez L, Vergés E, Gil F, et al. Cardioembolic stroke: risk factors, clinical features, and early outcome in 956 consecutive patients. Rev Invest Clin. 2020 May 7;73(5). doi: 10.24875/ RIC.20000227. Online ahead of print.

2. Campbell BCV, Khatri P. Stroke. Lancet. 2020;396:129-42.

3. Leifer D, Rundek T. Atrial cardiopathy: a new cause for stroke? Neurology. 2019;92:155-6.

4. Cerasuolo JO, Cipriano LE, Sposato LA. The complexity of atrial fibrillation newly diagnosed after ischemic stroke and transient ischemic attack: advances and uncertainties. Curr Opin Neurol. 2017;30:28-37.

5. Mavrogeni S, Koutsogeorgopoulou L, Dimitroulas T, Markousis-Mavrogenis G, Boki K, Katsifis G, et al. Combined brain/heart magnetic resonance imaging in systemic lupus erythematosus. Curr Cardiol Rev. 2020;16:178-86.

6. Calle-Botero E, Abril A. Lupus vasculitis. Curr Rheumatol Rep. 2020;22:71.

7. Behjati M, Sabri MR, Etemadi Far M, Nejati M. Cardiac complications in inherited mitochondrial diseases. Heart Fail Rev. 2020 Jul 29. doi: 10.1007/s10741-020-10009-1. Online ahead of print.

8. Lim A, Thomas RH. The mitochondrial epilepsies. Eur J Paediatr Neurol. 2020;24:47-52.

9. Rodríguez-Reyes H, Muñoz-Gutiérrez M, Salas-Pacheco JL. Current behavior of sudden cardiac arrest and sudden death. Arch Cardiol Mex. 2020;90:183-9.

10. Cronberg T, Greer DM, Lilja G, Moulaert V, Swindell P, Rossetti AO. Brain injury after cardiac arrest: from prognostication of comatose patients to rehabilitation. Lancet Neurol. 2020;19:611-22.

11. DeSimone CV, Graff-Radford J, El-Harasis MA, Rabinstein AA, Asirvatham SJ, Holmes DR. Cerebral amyloid angiopathy. J Am Coll Cardiol. 2017;70:1173-82.

12. Cortes-Canteli M, ladecola C. Alzheimer's disease and vascular aging: JACC Focus Seminar. J Am Coll Cardiol. 2020;75:942-51.

13. Yang M, Li C, Zhang Y, Ren J. Interrelationship between Alzheimer's disease and cardiac dysfunction: the brain-heart continuum? Acta Biochim Biophys Sin. 2020;52:1-8.

14. Sepehri Shamloo A, Dagres N, Müssigbrodt A, Stauber A, Kircher S, Richter S, et al. Atrial fibrillation and cognitive impairment: new insights and future directions. Heart Lung Circ. 2020;29:69-85.

15. Diener H-C, Hart RG, Koudstaal PJ, Lane DA, Lip GYH. Atrial fibrillation and cognitive function: JACC Review Topic of the Week. J Am Coll Cardiol. 2019;73:612-9.

16. Hachinski V. Dementia: paradigm shifting into high gear. Alzheimers Dement. 2019;15:985-94

17. Zamora Z, Williamson JD. The effects of lower BP goals on cognitive function in the elderly. Curr Cardiol Rep. 2020;22:63.

18. Rist PM, Buring JE, Ridker PM, Kase CS, Kurth T, Rexrode KM. Lipid levels and the risk of hemorrhagic stroke among women. Neurology. 2019;92:e2286-94.

19. Judge C, Ruttledge S, Costello M, Murphy R, Loughlin E, Álvarez-Iglesias A et al. Lipid Lowering Therapy, Low-Density Lipoprotein Level and Risk of intracerebral hemorrhage - a meta-analysis. J Stroke Cerebrovasc Dis. 2019;28:1703-9.

20. Alkhouli M, Graff-Radford J, Holmes DR. The heart-brain team - towards optimal team-based coordinated care. JAMA Cardiol. 2018;3:187. 